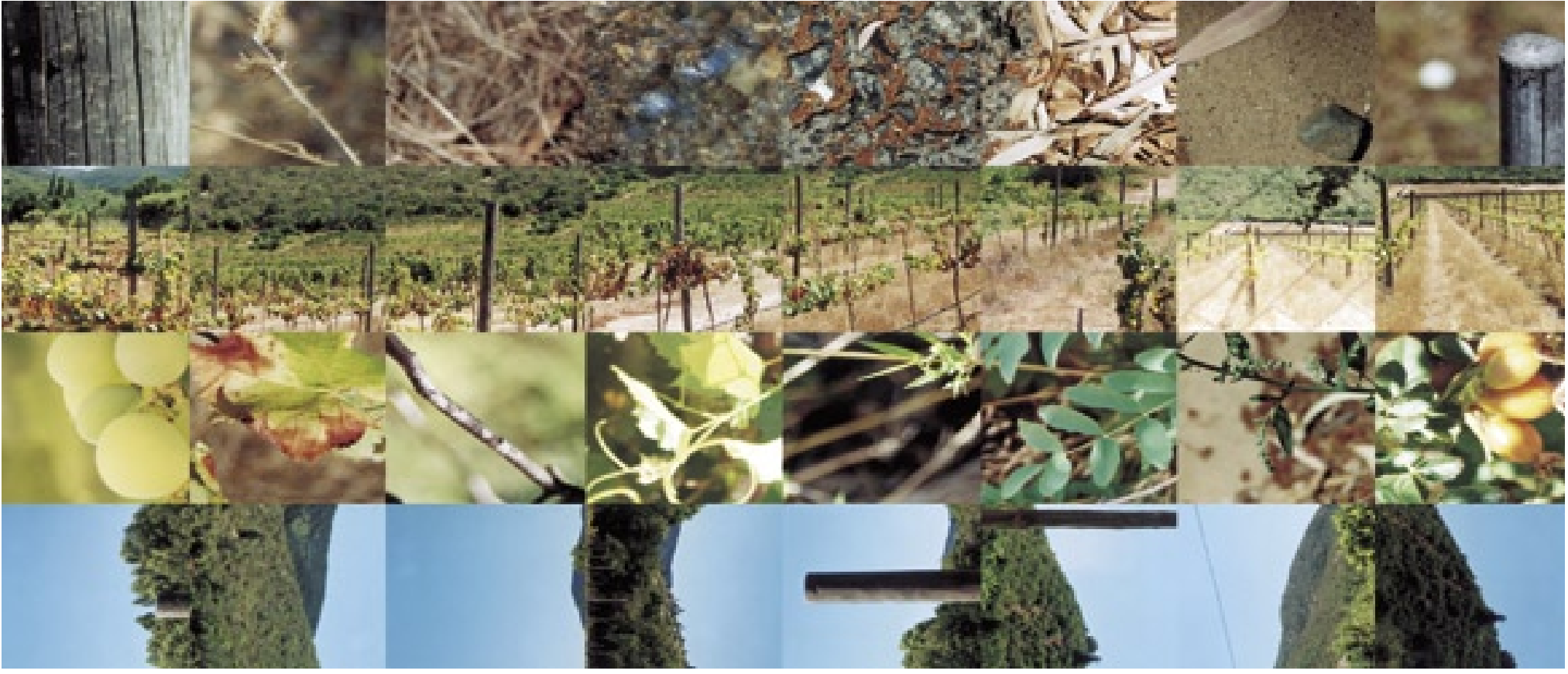

Texto: Teodoro Fernández L.

Un ambiente climático amable, de recursos y tecnologías limitadas, es el lugar de exploración de este taller conjunto entre las escuelas de arquitectura de la Pontificia Universidad Católica de Chile y de la Universidad Ricardo Palma de Lima. Se trata del desarrollo de un sistema constructivo para levantar lugares de sombra en tres viñas al sur de Santiago, a partir de piezas de madera de pequeñas dimensiones tratadas con técnicas elementales de ensamble.

Palabras clave: Estructuras de madera, sombreaderos, estructuras moduladas.

A world of limited resources and technologies in a friendly climate is explored in this joint workshop between the Pontificia Universidad Católica in Chile and Lima's Universidad Ricardo Palma. The challenge was to develop a constructive system to create shade in three vineyards south of Santiago, using wood in small dimensions and simple assembly techniques.

Key words: Wooden structures, pergolas, modular structures.

\title{
Cubiertas para viñedos
}

\section{Taller Perú - Verano 2003}

El que denominamos Taller Perú se desarrolló como taller de verano en la Escuela de Arquitectura de la Pontificia Universidad Católica de Chile durante los meses de enero, febrero y marzo de 2003. El equipo de profesores estuvo formado por los arquitectos Teodoro Fernández, Pilar García y Paulina Courard, en Chile, y René Poggione con Susana Biondi en Perú.

La posibilidad de establecer contactos personales entre alumnos y profesores así como conocer la arquitectura de la costa del Perú y del valle central de Chile a través de la realización de diferentes proyectos fueron temas implícitos del taller.

El taller se desarrolló como un convenio con la Escuela de Arquitectura de la Universidad Ricardo Palma de Lima, gracias a la dedicación y entusiasmo de los profesores arquitectos René Poggione y Susana Biondi, y un grupo de dieciséis alumnos limeños en conjunto con catorce alumnos chilenos.

El taller trata de centrar la discusión en los aspectos formales de la construcción en madera: -Una persecución y búsqueda desde la forma como objetivo central, que informa constantemente lo que se quiere lograr a través de la invención estética y tecnológica de los aspectos constructivos de la forma final.

-Una investigación sobre aspectos constructivos y formales de la madera en dimensiones pequeñas y medianas, con recursos habituales, elaborando un sistema constructivo capaz de adecuarse a diferentes programas y localizaciones, con técnicas artesanales o aquellas que podemos llamar de baja tecnología.

Se recurre a los que sin pudor, al contrario de los arquitectos, buscan en los aspectos formales la transformación de los materiales y la materia: los artistas del siglo XX. Se ha seleccionado un grupo de ellos: Naum Gabo, Richard Serra, Jorge Oteíza, Martin Puryear, Sol Lewitt, Alexander Calder, Anthony Caro, investigando en sus búsquedas las posibilidades de transformación de un material como la madera elaborada directamente en la consecución de resultados formales.

Función, programa y lugar se revelan a través de una forma preconcebida que se integra en el tiempo y propone al lugar un nuevo modo de expresarse, a la manera de los estilos tradicionales en el arte. Con los sistemas constructivos inventados en el taller se realizaron dos anteproyectos: uno en diversas viñas en el valle central de Chile y otro en ruinas o sitios arqueológicos de la costa peruana. ARQ 


\section{Propuesta para viña en Chimbarongo}

Mauricio Magnasco + Raimundo Morales

El proyecto se basa en el trabajo de Anthony Caro. Estas esculturas eran concebidas como la adición de objetos reciclados y descontextualizados. Siguiendo la lógica del artista, buscamos materiales en demoliciones y elaboramos un catálogo de elementos.

En la etapa de desarrollo exploramos las posibles combinaciones para conformar un espacio manteniendo los principios del artista. Destacaron algunos elementos por sobre otros por las posibilidades constructivas que ofrecían. El pilar octogonal fue uno, que al articularlo con barras de 6 metros en ángulos de $45^{\circ}$ y $90^{\circ}$, nos permitió armar una estructura con tres puntos de apoyo que sostenía una serie de cubiertas articuladas, hechas con puertas y ventanas.
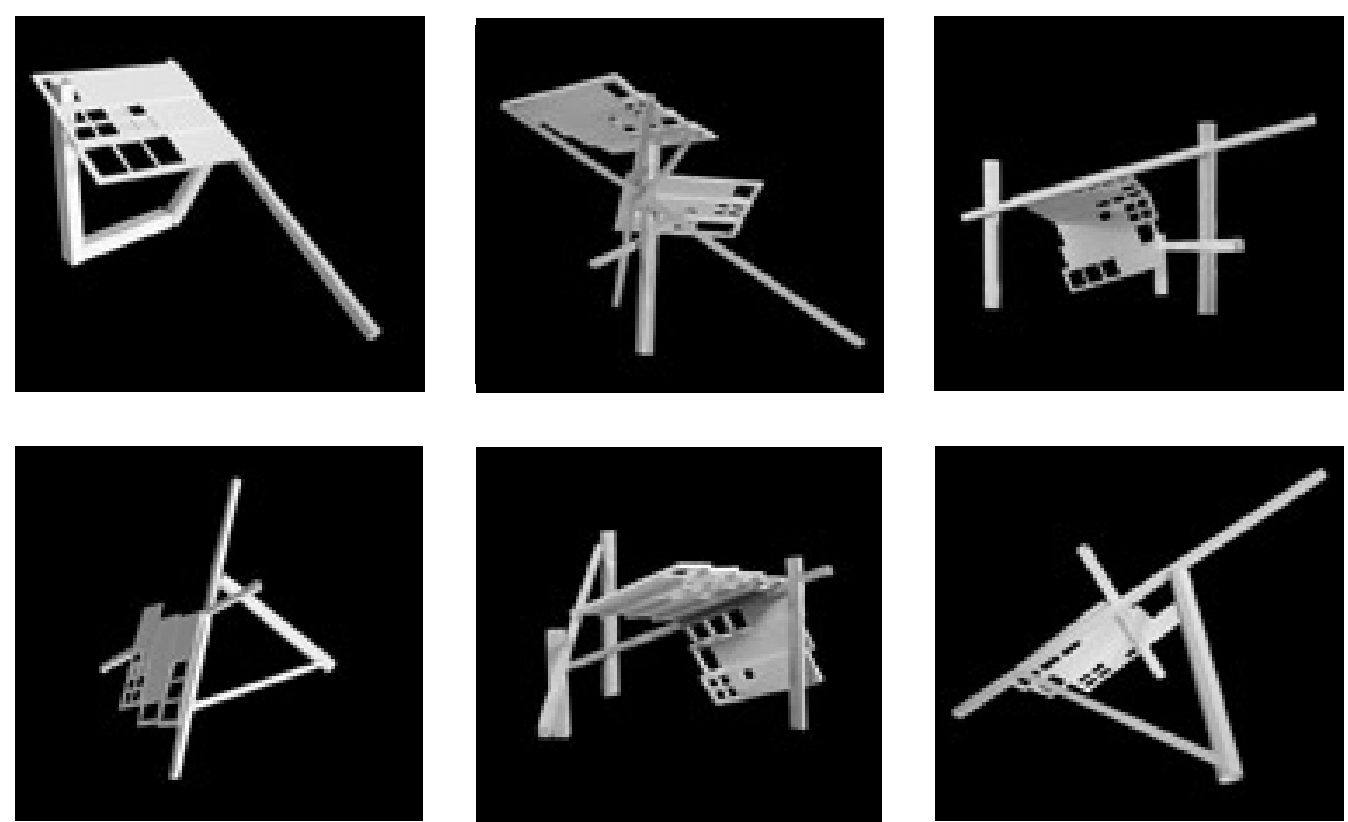

Chile
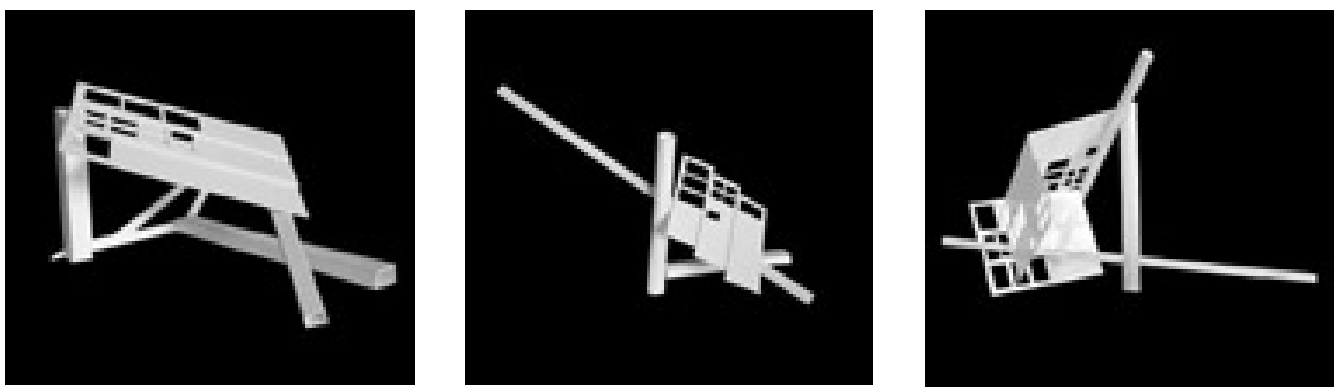

Combinatorias a partir de elementos de demolición

Mauricio Magnasco + Raimundo Morales +

María José Sánchez + Paula Orta +

Soledad Poehler + Daniel Talesnik +

Daniel Valenzuela + Rafael Zamora.

Perú

Profesores René Posgione + Susana Biondi

Estudiantes Sandro Angobaldo +

Carlos Echegaray + Sebastián La Rosa +

Michelle Llona + Rodrizo Martínez +

Patricia Melgarejo + Fernando Moschera +

José Pacheco + Chiqui Palacios + Ximena Peña +

Jorge Sánchez + Carola Soto +

Marianne Tapia + Camilo Valdivia 
Propuestas para viña

en Nancagua

Fernando Colchero + María José Sánchez Tomando como referente elementos del trabajo del escultor vasco Jorge Oteíza, se diseñó un sistema constructivo en madera a partir del ensamble de planchas de terciado, unidas en ángulo de $60^{\circ}$. El sistema consiste en una cubierta que funciona en base a piezas diagonales $\mathrm{y}$ horizontales, las cuales se entrelazan para generar una malla estructural. Esta cubierta se sustenta sobre pilares y produce juegos de luz y sombra, en forma similar a los parrones de las viñas. Situándose en el límite entre un bosque de árboles antiguos y los parronales de la viña, el pabellón para visitantes se plantea como un elemento de articulación entre ambos, el cual permite disfrutar de la sombra del bosque y la vista sobre las viñas.
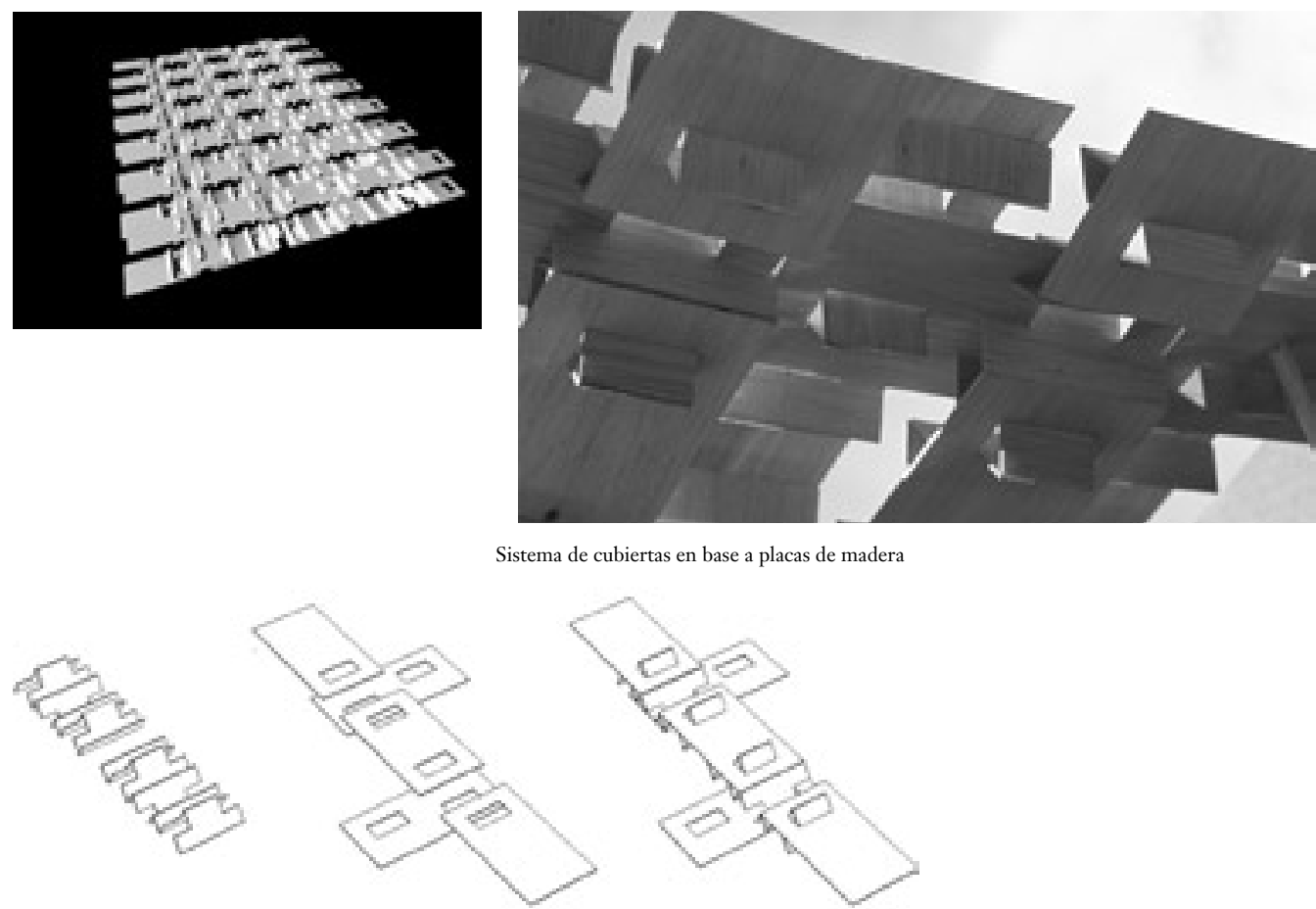

Axonométrica de elementos. Placas diagonales y horizontales encajadas
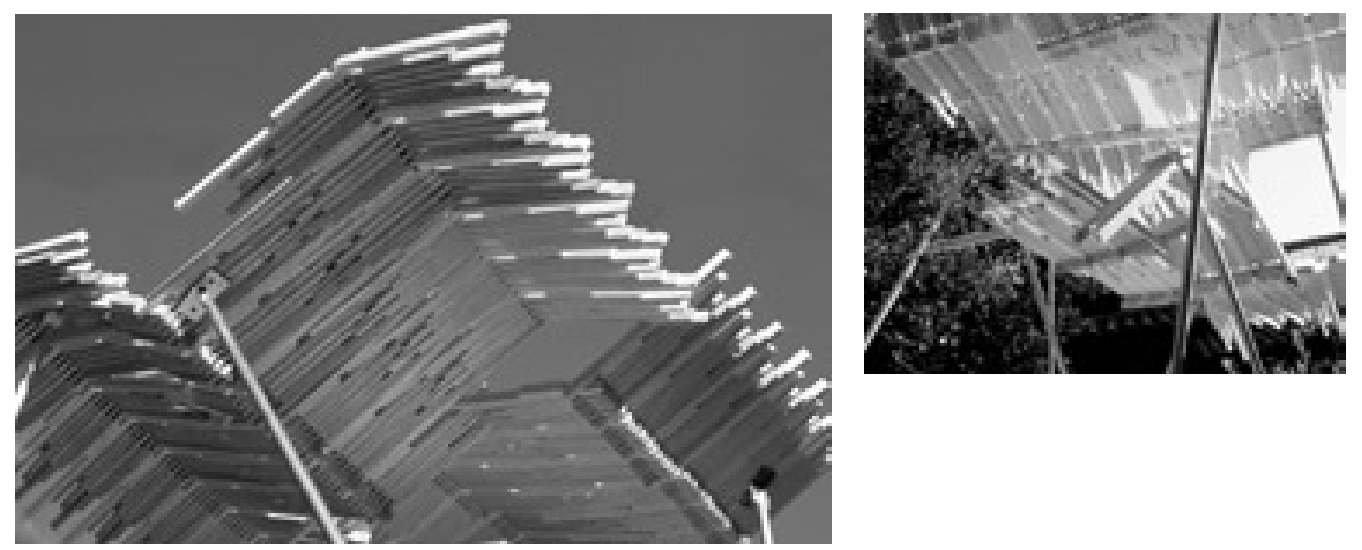

Paula Orta + Soledad Poehler

Paneles laminados a partir de Martin Puryear: un sistema compuesto por paneles autosoportantes de listones de distinto largo que forman un endentado. Éste permite múltiples maneras de unión además de la posibilidad de controlar luz y ventilación. Los paneles pueden ser usados para resolver paramentos o cubiertas: en ambos casos, se unen entre sí a través de piezas y pernos de acero.
Combinatoria en sistema de paneles a partir de tablas y listones
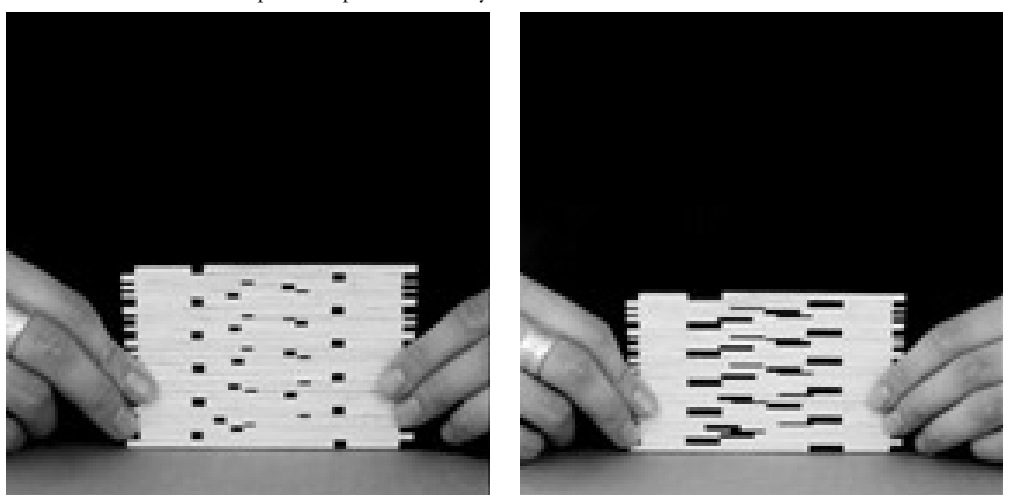
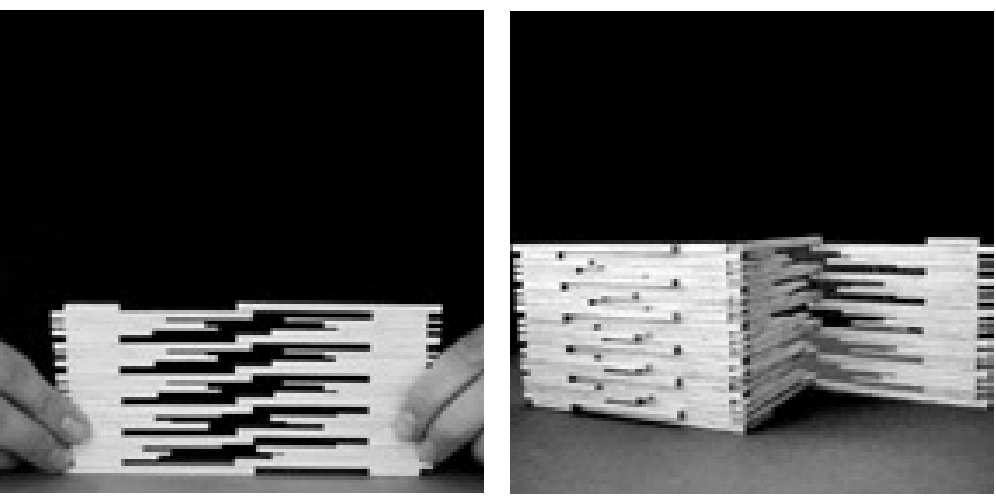
Daniel Talesnik + Rafael Zamora + Rodrigo Martínez

Se trabajó un comedor, una cocina, baños y una terraza para recibir a los turistas en la viña. Es la etapa intermedia del desarrollo de un sistema constructivo que nace a partir del estudio de la obra escultórica de Naum Gabo. El anteproyecto se monta en unas pircas construidas en una pequeña quebrada de un río que cruza la viña. Se ocupan tres tipos diferentes de cerchas de madera para individualizar los sectores en la propuesta. Estando dentro de las instalaciones el espacio se ve ritmado por las cerchas; esto se relaciona con la disposición de las hileras de parras en la viña.
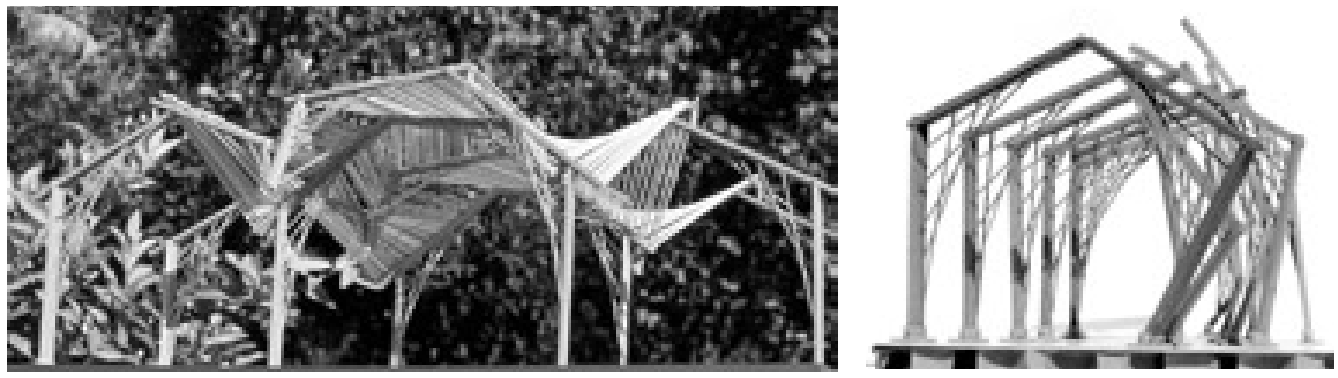

Vista de maqueta, sombreader
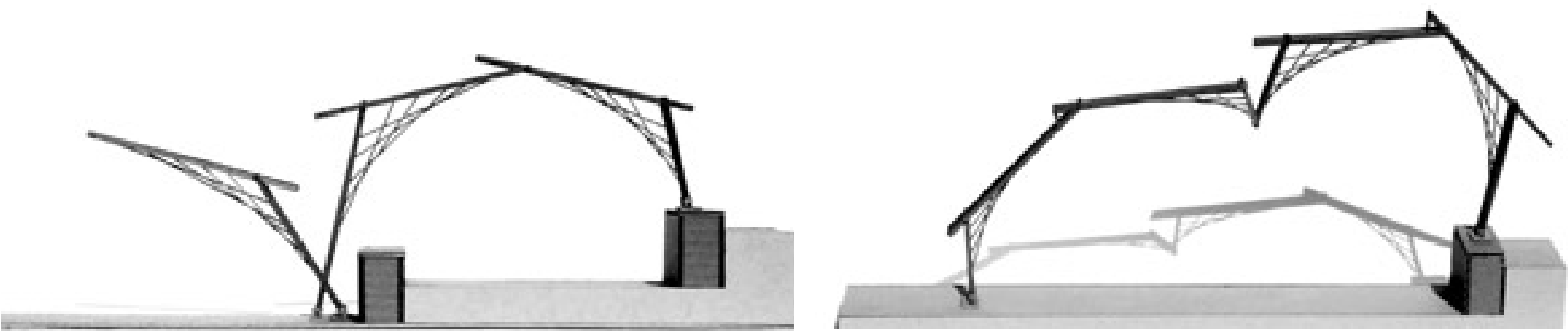

Variantes de sistema de cerchas, combinables entre sí

\section{Andrés Berr + Héctor Colonelli}

Partiendo del concepto de láminas apoyadas en equilibrio, del escultor norteamericano Richard Serra y del efecto repetitivo y direccionador de las líneas de plantación de los viñedos, generamos un sistema estructural por repetición de paneles finger joint de $100 \times 350 \times 6 \mathrm{~cm}$, unidos por un tubular de acero que a la vez sostiene las vigas que, igualmente, son módulos de éste.

Los tableros dan la sensación de inestabilidad y movimiento de la estructura al estar inclinados o plegados; el pliegue rigidiza el sistema y lo vuelve autosoportante.

El pabellón se ubica al borde de la quebrada que atraviesa la viña, bajo unos eucaliptus. Enfrenta los mismos viñedos que inspiran su forma.
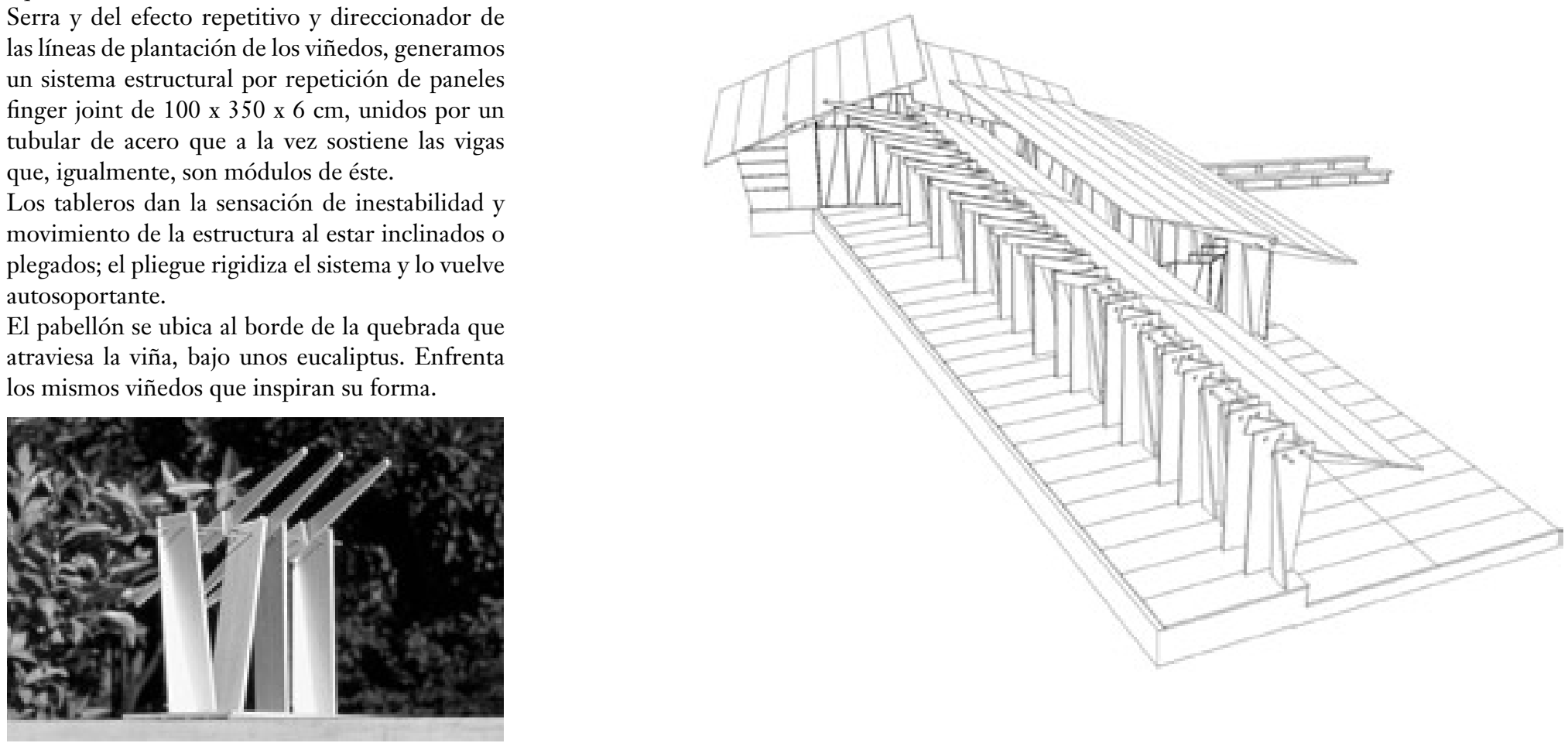

Vista de maqueta, sistema estructural 